\title{
Estudio para mejorar la adherencia al tratamiento del paciente adulto joven con VIH/SIDA según la teoría del autocuidado
}

\author{
Study to Improve Adherence to the Treatment of Young Patients with HIV / AIDS According to \\ Self-Care Deficit Theory
}

\author{
Arias-Rico $J^{a}$, Jiménez-Sánchez $R C^{b}$, Rivera-Ramírez $L A{ }^{c}$, Cervantes-Yautenzi MR ${ }^{d}$, \\ Cervantes-Ortega $L^{e}$, Monter-Acosta $D^{e}$,Delgado-Serrano A ${ }^{\mathrm{e}}$, Bustamante-Mejía $S{ }^{\text {e }}$, \\ Burgos- Montiel A ${ }^{e}$, Fragoso-Aguilar $E^{e}$
}

\begin{abstract}
:
The World Health Organization defines the adherence to treatment as taking the medication according to the dosage in the prescribed program and the persistence as well as throughout the indicated time. At present the detachment to long-term treatments is a problem that out-regulates the patient's health. The aim was to identify which nursing interventions improve adherence to the treatment of young adult patients with HIV / AIDS. Methodology: This is a descriptive quantitative study applied to a sample of 30 patients from a Care Center for HIV / AIDS Patients. Results: According to the results obtained, adherence to the compliance treatment is found with $36.7 \%$. The variable of assistance to consultations frequently showed $56.7 \%$ and never with $3.3 \%$ and the use of condoms has a percentage of $60 \%$ in always being the most frequent. Discussion: It was found that interventions are not relevant when abandonment is usually due to economic, family factors and difficult access to health systems that tend to be the most frequent. Conclusion: The results of the investigation showed that the prevailing factors that intervene in adherence to the treatment of patients with HIV / AIDS are economic, emotional management, inadequate intake of treatment and periodic non-attendance at consultations. Nursing interventions that improved adherence to treatment were education, disease information and emotional support.
\end{abstract}

Keywords:

Quality of life, pharmacological treatment, adherence to treatment, nursing

Resumen:

Introducción: La Organización Mundial de la Salud define la adherencia al tratamiento como tomar la medicación de acuerdo a la dosificación en el programa prescrito y la persistencia así como a lo largo del tiempo indicado. En la actualidad el desapego a los tratamientos a largo plazo es un problema que descontrola la salud del paciente. El objetivo fue identificar qué intervenciones de enfermería mejoran la adherencia al tratamiento del paciente adulto joven con VIH/SIDA. Metodología: Es un estudio cuantitativo de carácter descriptivo aplicado a una muestra de 30 pacientes de un Centro de Atención para Pacientes con VIH/SIDA. Resultados: De acuerdo con los resultados obtenidos se encuentra una adherencia al tratamiento de cumplimiento con un $36.7 \%$. La variable de asistencia a consultas arrojó frecuentemente con un $56.7 \%$ y nunca con el $3.3 \%$ y el uso de preservativo tiene un porcentaje de $60 \%$ en siempre siendo está la más frecuente. Discusión: Se encontró que las intervenciones no tienen relevancia cuando el abandono suele deberse a factores económicos, familiares y del difícil acceso a los sistemas de salud que suelen ser los que más se presentan. Conclusión: Los resultados de la investigación arrojaron que los factores prevalentes que intervienen en la adherencia al tratamiento de los pacientes con VIH/SIDA son de tipo económico, manejo de emociones, ingesta inadecuada del tratamiento y la inasistencia periódica a las consultas. Las intervenciones de enfermería que mejoraron la adherencia al tratamiento, fueron la educación, la información de la enfermedad y el apoyo emocional.

\section{Palabras Clave:}

Calidad de vida, tratamiento farmacológico, adherencia al tratamiento, enfermería 


\section{Introducción}

La Organización Mundial de la Salud (OMS) define la adherencia al tratamiento como el cumplimiento del mismo; es decir, tomar la medicación de acuerdo con la dosificación del programa prescrito; y la persistencia, tomar la medicación a lo largo del tiempo [1]. Uno de los problemas que surge en esta enfermedad es que el paciente no se siente preparado para enfrentar esta situación. El incumplimiento del tratamiento es la principal causa de que no se obtengan todos los beneficios que los medicamentos pueden proporcionar a los pacientes, está en el origen de complicaciones médicas y psicosociales de la enfermedad, reduce la calidad de vida de los pacientes, aumenta la probabilidad de aparición de resistencia a los fármacos y desperdicia recursos asistenciales[2].

La persona es un todo integral dinámico que funciona biológicamente, simbólicamente y socialmente, con la facultad de utilizar las ideas, las palabras para pensar y reflexionar sobre su estado de salud, guiar sus esfuerzos a fin de llevar a cabo acciones de autocuidado y el cuidado dependiente [3]. El efecto que tiene la adherencia en cualquier fase de la enfermedad satisfaciendo las necesidades físicas, emocionales, espirituales y sociales del enfermo. Enfermería a través del tiempo ha logrado tener una buena influencia a través de las intervenciones realizadas para el mejoramiento de la adherencia al tratamiento. Los factores relacionados con la adherencia al tratamiento antirretroviral proceden de las características individuales, por eso la importancia de brindar información. Como recomendaciones se sugieren, entre otras, actividades de educación para el autocuidado y talleres para la mayor comprensión de la enfermedad así como para mejorar su autoestima, su empoderamiento y la aceptación de sí mismos. De esta manera será posible medir en el paciente adulto joven cuáles son las mejores técnicas de intervención para que éste se adhiera al tratamiento y su calidad de vida mejore [4]. Sus necesidades están en directa relación con su situación psicosocial así como con el cuidado de su salud. El objetivo fue identificar los factores que intervienen en la adherencia al tratamiento según la teoría del Déficit del Autocuidado de Dorotea Orem de los adultos jóvenes diagnosticados con VIH/SIDA, identificando los factores condicionantes básicos que más influyen sobre la adherencia y de la misma manera realizar las intervenciones de enfermería que mejor resultado tienen, todo esto se realizó en un Centro Especializado en $\mathrm{VIH} / \mathrm{SIDA}$ de Hidalgo.

\section{Metodología}

Se utilizó un instrumento de valoración que nos permite diferenciar los factores que intervienen en la adherencia al tratamiento con VIH/SIDA de estos pacientes, el instrumento de valoración cuenta con veinticinco ítems clasificados por áreas de identificación personal, apoyo emocional, apoyo económico, y educación sexual. En la investigación realizada los recursos humanos fueron: Seis responsables de la investigación, treinta personas encuestadas que cumplían con los criterios de inclusión, cuatro asesores de tesis. $Y$ los recursos materiales utilizados, fueron: Instrumento de evaluación (encuestas), apegándose a los criterios que el Comité de Ética del Instituto de Ciencias de la Salud exige como es el consentimiento informado, cuidando la integridad y anonimato de los participantes.

\section{Resultados}

Se aplicó la encuesta a 30 personas identificadas dentro de un hábitat clínico $(\mathrm{VIH}), 18$ masculinos y 12 femeninos. Dentro del rango de edad predomina de 31 a 40 años en un $56.8 \%$, mostrando un porcentaje inferior el rango de 20-30 con un $43.2 \%$ del total. La variable Escolaridad arroja un predominio doble, siendo el nivel Secundaria y Bachillerato representando un $33.3 \%$, el grado Universitario representa un $23.3 \%$, el restante obtuvo mínimo Primaria. La variable Estado Civil arroja que el $43.3 \%$ de la población (n) se encuentra Soltero(a), Unión libre $30 \%$ y Casado con una frecuencia menor. En la variable Nivel socioeconómico se obtuvo que un $83.3 \%$ posee un nivel medio, un nivel Bajo un $10 \%$ y el resto poseen un estatus económico Alto. En la variable Religión que profesa la población (n) se encuentra mayor porcentaje en la religión Católica con el $60 \%$, Cristiana $20 \%$, Otro(a) $16.7 \%$ y Testigo de jehová frecuencia única. En la variable Comparte domicilio se encontró Compartido con $56.7 \%$, el resto Independiente. El tiempo activo de VIH/SIDA representa mayor prevalencia el rango de 0 a 3 años con $43.3 \%, 4$ a 7 años un $30 \%$ y de 8 a 11 años un $28.7 \%$ de la población (n). La variable Familiares Directos activos con VIH/SIDA representa mayor prevalencia Ninguno con $60 \%$, Pareja con $36.7 \%$, Padres en frecuencia única. En Actividad Física (frecuencia) se encontró mayor prevalencia en "Pocas veces" con un $50 \%$, "Frecuente" con $36.7 \%$, "Nunca" $10 \%$ y "Siempre" escaso (Tabla 1).

Tabla 1. Datos sociodemográficos de los sujetos participantes.

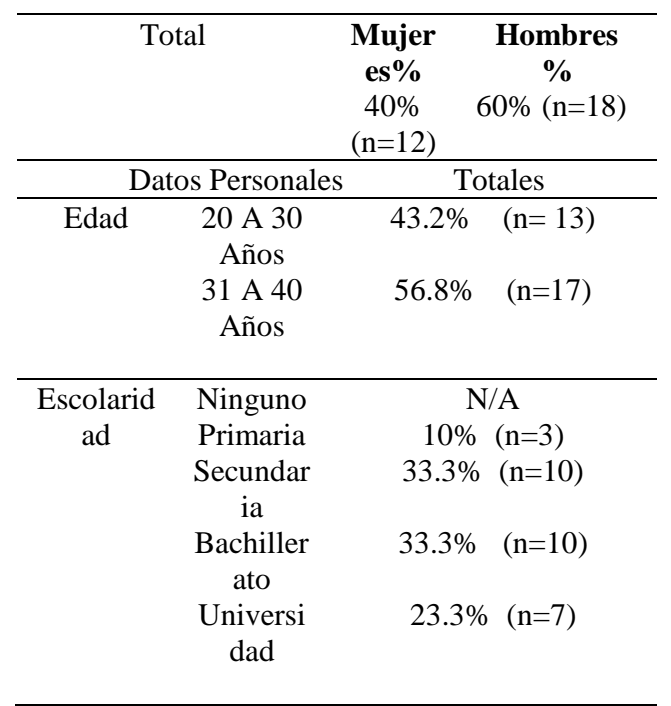




\begin{tabular}{|c|c|c|}
\hline \multirow{5}{*}{$\begin{array}{l}\text { Estado } \\
\text { Civil }\end{array}$} & Soltero & $43.3 \% \quad(n=13)$ \\
\hline & Casado & $26.6 \% \quad(n=8)$ \\
\hline & Unión & $30 \% \quad(n=9)$ \\
\hline & Libre & \\
\hline & Viudo & N/A \\
\hline \multirow[t]{3}{*}{$\begin{array}{l}\text { Preferenc } \\
\text { ia Sexual }\end{array}$} & $\begin{array}{c}\text { Heterose } \\
\text { xual }\end{array}$ & $56.7 \% \quad(\mathrm{n}=17)$ \\
\hline & $\begin{array}{c}\text { Homose } \\
\text { xual }\end{array}$ & $33.3 \% \quad(n=10)$ \\
\hline & Bisexual & $10 \% \quad(n=3)$ \\
\hline \multirow{3}{*}{$\begin{array}{c}\text { Nivel } \\
\text { Socioeco } \\
\text { nómico }\end{array}$} & Bajo & $10 \%(n=3)$ \\
\hline & Medio & $83.3 \% \quad(n=25)$ \\
\hline & Alto & $6.7 \% \quad(n=2)$ \\
\hline \multirow[t]{6}{*}{ Religión } & Católica & $60 \% \quad(n=18)$ \\
\hline & Cristiana & $20 \%(n=6)$ \\
\hline & Testigo & $3.3 \%(n=1)$ \\
\hline & & \\
\hline & Jehová & \\
\hline & Otro & $16.7 \%(n=5)$ \\
\hline \multirow{4}{*}{$\begin{array}{c}\text { Compart } \\
\mathrm{e} \\
\text { Domicili } \\
\mathrm{o}\end{array}$} & Sí & $56.7 \%(\mathrm{n}=17)$ \\
\hline & No & $53.3 \% \quad(n=13)$ \\
\hline & & \\
\hline & & \\
\hline \multirow{8}{*}{$\begin{array}{l}\text { Tiempo } \\
\text { Con Vih }\end{array}$} & $0-3$ & $43.3 \% \quad(n=13)$ \\
\hline & Años & \\
\hline & $4-7$ & $30 \%(n=9)$ \\
\hline & Años & \\
\hline & $8-11$ & $26.7 \%(\mathrm{n}=8)$ \\
\hline & Años & \\
\hline & Más De & N/A \\
\hline & 12 Años & \\
\hline \multirow{4}{*}{$\begin{array}{c}\text { Familiare } \\
\text { s Con } \\
\text { Vih }\end{array}$} & Ninguno & $60 \%(\mathrm{n}=18)$ \\
\hline & Padres & $3.3 \%(\mathrm{n}=1)$ \\
\hline & Pareja & $36.7 \%(\mathrm{n}=11)$ \\
\hline & Hijos & N/A \\
\hline \multirow{4}{*}{$\begin{array}{l}\text { Activida } \\
\text { d Física }\end{array}$} & Nunca & $10 \%(\mathrm{n}=3)$ \\
\hline & Pocas & $50 \%(n=15)$ \\
\hline & Veces & \\
\hline & $\begin{array}{c}\text { Frecuent } \\
\text { emente }\end{array}$ & $36.7 \%(\mathrm{n}=11)$ \\
\hline
\end{tabular}

El manejo de emociones, con $43.3 \%$ a pocas veces y un $10 \%$ nunca. El consumo de alcohol refirió que frecuentemente tiene un porcentaje de $33.2 \%$, pocas veces $36.7 \%$ y una mínima en nunca con un porcentaje de $30 \%$. La variable del consumo de drogas presenta pocas veces con un porcentaje de $20 \%$, nunca con $70 \%$. La variable que presenta el uso de preservativo tiene un porcentaje de $60 \%$ en siempre siendo está la más frecuente.
En la variable de la persona que lo apoya económicamente tiene un $85.7 \%$ en que lo apoyan sus padres, un $14.3 \%$ pareja. En la persona que lo apoya emocionalmente, tiene mayor porcentaje pareja e hijos con $40 \%$ y padres con $26.7 \%$. La variable de conocimiento familiar sobre la enfermedad arrojó con mayor frecuencia el sí con un $76.7 \%$, no un $23.3 \%$. En la variable de adherencia al tratamiento arrojo, de $0-25 \%$ de cumplimiento un $25 \%$ un $3.3 \%$, y de $76 \%-100 \%$ de cumplimiento un $36.7 \%$. La variable de asistencia a consultas arrojó, frecuentemente $56.7 \%$ y nunca un $3.3 \%$ (Tabla 2).

Tabla 2. Distribución de las diferentes variables estudiadas de los sujetos participantes.

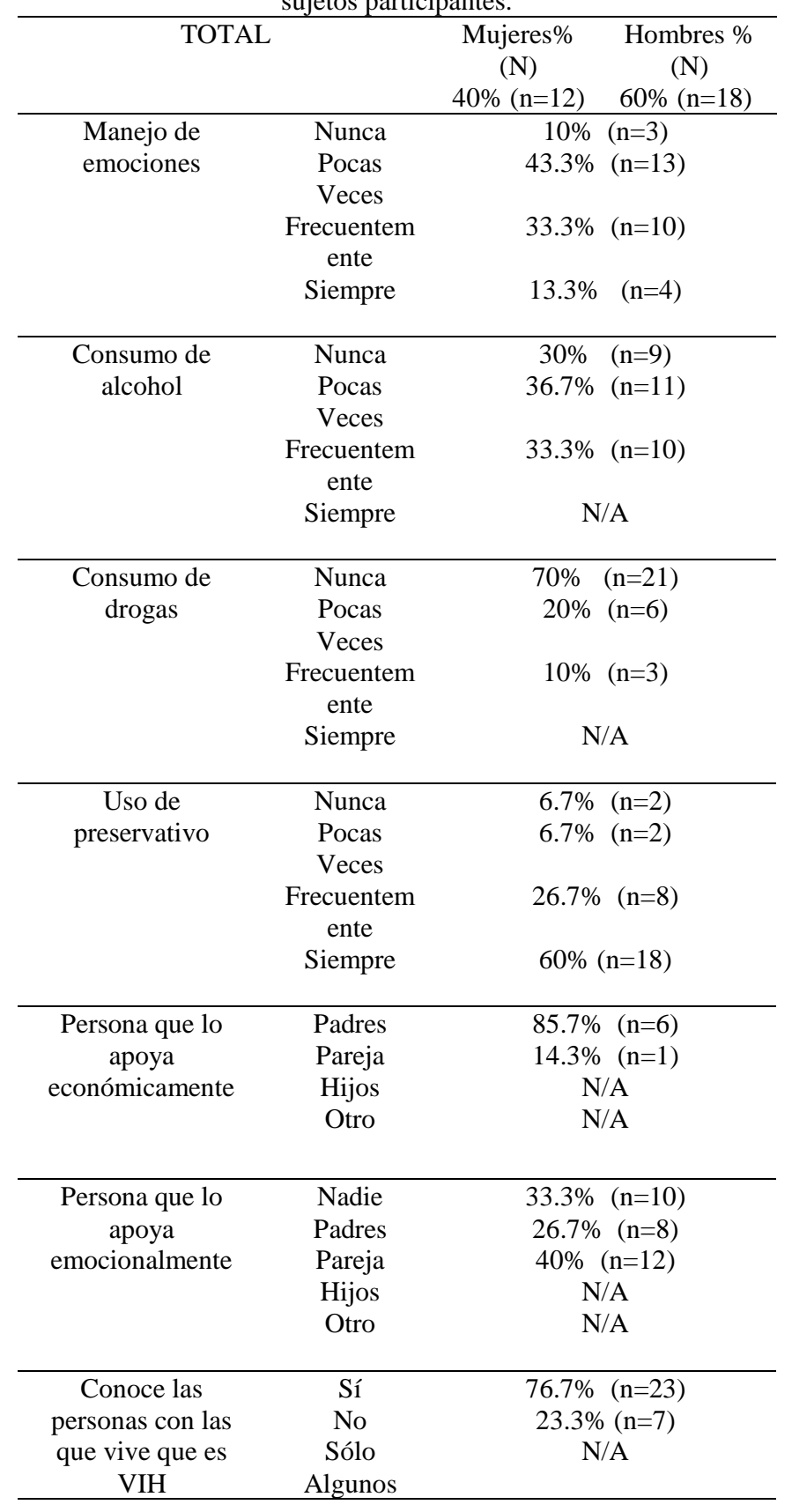




\begin{tabular}{ccc}
\hline $\begin{array}{c}\text { Cumple al 100\% } \\
\text { con el tratamiento } \\
\text { contra el VIH }\end{array}$ & $0-25 \%$ & $3.3 \%(\mathrm{n}=1)$ \\
& $26-50 \%$ & $26.7 \%(\mathrm{n}=8)$ \\
& $51-75 \%$ & $33.3 \%(\mathrm{n}=10)$ \\
& $76-100 \%$ & $36.7 \%(\mathrm{n}=11)$ \\
\hline $\begin{array}{c}\text { Asiste a todas sus } \\
\text { consultas que } \\
\text { tienen relación con } \\
\text { el VIH }\end{array}$ & $\begin{array}{c}\text { Nunca } \\
\text { Frecuentem }\end{array}$ & $\begin{array}{c}\text { ente } \\
\text { Siempre }\end{array}$ \\
\hline & & $56.7 \%(\mathrm{n}=1)$ \\
& & Fte. IMPVATPCVIH/SIDA2018
\end{tabular}

\section{Discusión}

La prevalencia y los factores asociados a la no adherencia al tratamiento en adultos infectados con el VIH/SIDA; se publicaron datos donde la edad promedio fue de 36.8 años; $64.1 \%$ era del sexo masculino y el $35.9 \%$ de los encuestados resultó ser no adherente [5]; a diferencia de esta investigación ya que la edad promedio fue de 30 años de edad; el $60 \%$ fue del sexo masculino y un $36,7 \%$ se adhiere completamente a su tratamiento.

Se instauró la relación entre la adherencia al tratamiento antirretroviral, situación clínica y detección de los factores que se relacionan con la falta de adherencia; en éste se recogieron variables sociodemográficas y otros factores que dificultan la toma de medicación e influían en el cumplimiento del tratamiento; a diferencia de la investigación realizada, el $63.3 \%$ se encuentra dentro del rango del 0 al $75 \%$ de la no adherencia al tratamiento, debido a factores como el manejo de emociones, el ámbito económico y el no tomar sus fármacos $[6,7]$.

La adherencia a aspectos no-farmacológicos del tratamiento en personas con $\mathrm{VIH} /$ Sida realizado en la ciudad de Cali, Colombia en el año 2015, un estudio observacional transversal, con una muestra de 277 personas con $\mathrm{VIH} /$ Sida de nueve instituciones de salud. Se utilizó el cuestionario de adherencia al tratamiento para el VIH/Sida. Sólo el $37 \%$ de las personas son adherentes al tratamiento no farmacológico. A diferencia de esta investigación manejo de emociones, se encontró con un $42.9 \%$ siempre, $28.6 \%$ frecuentemente, y con $14.3 \%$ a pocas veces. El consumo de alcohol refirió $42.9 \%$ pocas veces y nunca $28.6 \%$. Consumo de drogas presenta pocas veces con mayor frecuencia, con un $42.9 \%$, nunca con $28.6 \%$. La variable que presenta el uso de preservativo tiene un $57.1 \%$ siempre siendo está la más frecuente $[8,9]$.

Las características en los pacientes con abandono de terapia antirretroviral en el municipio Boyeros durante el 2013 y la descripción de sus consecuencias económicas. Se estudiaron 124 pacientes. Los datos fueron obtenidos de las tarjetas de control de medicamentos y las historias clínicas de la consulta municipal de Infectología. Se han publicado datos donde el 60,5 \% resultó abandono intermitente y $17 \%$ abandono total. Predominó el sexo masculino. A diferencia de esta investigación donde adherencia al tratamiento arrojo, de $0-25 \%$ de cumplimiento un $25 \%$ y de $76 \%-100 \%$ de cumplimiento un $36.7 \%[10,11]$.

\section{Conclusiones}

La adherencia al tratamiento del paciente adulto joven con VIH/SIDA se ve mejorada con la educación acerca de los tratamientos antirretrovirales, la información acerca de la enfermedad y el apoyo emocional. Los factores condicionantes básicos de acuerdo a la teoría de Orem que influyen en la adherencia al tratamiento del paciente con VIH/SIDA destacan notablemente la edad, orientación sociocultural, factores del sistema de cuidados de salud, factores del sistema familiar y la disponibilidad y adecuación de los recursos.

\section{Referencias}

[1].Gavidia, Haro, et al. Estrategias de afrontamiento y calidad de vida en pacientes diagnosticados con VIH en el Hospital Provincial General Docente Riobamba, período octubre 2015-marzo 2016. BS thesis. Riobamba: Universidad Nacional de Chimborazo, 2016.

[2].Herrera Díaz, María José, et al. Evaluación del cumplimiento de la guía de atención en pacientes con VIH del Distrito de Barranquilla diagnosticados en el periodo de enero a septiembre 2017. 2018. Tesis de Maestría. Universidad del Norte.

[3].Naranjo Hernández, Ydalsys, et al. "La teoría Déficit de autocuidado: Dorothea Elizabeth Orem." Gaceta Médica Espirituana 19.3 (2017): 89-100.

[4].Hernández, Ydalsys Naranjo, José Alejandro Concepción Pacheco, and Miriam Rodríguez Larreynaga. "La teoría Déficit de autocuidado: Dorothea Elizabeth Orem." Gaceta Médica Espirituana 19.3 (2018).

[5].Córdova Alarcón, Sonia Birmania, Gualotuña Alcívar, and Cinthya Lizeth. "'Identificación del porcentaje de pacientes adultos mayores con mala adherencia al tratamiento antihipertensivo y los factores asociados en la consulta externa de medicina interna de un hospital público de la ciudad de Quito en el primer semestre del 2018"." (2018).

[6].Rodríguez Quesada, Pedro Pablo. Estudio de factores de riesgo influyentes en el adherencia al tratamiento antirretroviral y desarrollo de un modelo predictivo en pacientes VIH positivos. Diss. Universidad Complutense de Madrid, 2016.

[7].Sánchez-Rivero, Irene, et al. "Influencia del consumo de sustancias y el deterioro cognitivo en la adherencia al tratamiento antirretroviral en pacientes VIH+." Adicciones(2018).

[8].Benavides Ponce, Magda Fernanda. "Adherencia al tratamiento y autoeficacia en hombres con VIH/Sida de la ciudad de Cali." (2017).

[9].Suárez-Villa, Mariela, et al. "Adherencia a fármaco-terapia antirretroviral para el tratamiento del VIH/SIDA en la costa caribe colombiana." Revista Latinoamericana de Hipertension 13.2 (2018): 20-25.

[10]. Olivares Nunura, José Luis. "Factores clínicos y epidemiológicos más frecuentes de los pacientes con diagnóstico de VIH/SIDA atendidos en el Hospital de Apoyo I Nuestra Señora de las Mercedes-Paita durante el periodo julio 2016-Junio 2017." (2018).

[11]. Glocer, Dina, et al. "Motivos de Internación en Pacientes HIV/SIDA en un Hospital de Segundo Nivel de Complejidad de la ciudad de Rosario." 2013. 